\title{
Four mutations in MITF, SOX10 and PAX3 genes were identified as genetic causes of waardenburg syndrome in four unrelated Iranian patients: case report
}

Safoura Zardadi ${ }^{1}$, Sima Rayat ${ }^{1}$, Maryam Hassani Doabsari ${ }^{2}$, Aliagha Alishiri ${ }^{3}$, Mohammad Keramatipour $^{4}$, Zeynab Javanfekr Shahri ${ }^{5}$ and Saeid Morovvati ${ }^{* *}$

\begin{abstract}
Background: Waardenburg syndrome (WS) is a rare genetic disorder. The purpose of this study was to investigate clinical and molecular characteristics of WS in four probands from four different Iranian families.

Case presentation: The first patient was a 1-year-old symptomatic boy with congenital hearing loss and heterochromia iridis with a blue segment in his left iris. The second case was a 1.5-year-old symptomatic girl who manifested congenital profound hearing loss, brilliant blue eyes, and skin hypopigmentation on the abdominal region at birth time. The third patient was an 8-month-old symptomatic boy with developmental delay, mild atrophy, hypotonia, brilliant blue eyes, skin hypopigmentation on her hand and foot, Hirschsprung disease, and congenital profound hearing loss; the fourth patient was a 4-year-old symptomatic boy who showed dystopia canthorum, broad nasal root, synophrys, skin hypopigmentation on her hand and abdomen, brilliant blue eyes, and congenital profound hearing loss. Whole exome sequencing (WES) was used for each proband to identify the underlying genetic factor. Sanger sequencing was performed for validation of the identified mutations in probands and the available family members. A novel heterozygous frameshift mutation, c.996delT (p.K334Sfs*15), on exon 8 of the MITF gene was identified in the patient of the first family diagnosed with WS2A. Two novel de novo heterozygous mutations including a missense mutation, c.950G > A (p.R317K), on exon 8 of the MITF gene, and a frameshift mutation, c.684delC (p.E229Sfs*57), on the exon 3 of the SOX10 gene were detected in patients of the second and third families with WS2A and PCWH (Peripheral demyelinating neuropathy, Central dysmyelinating leukodystrophy, Waardenburg syndrome, Hirschsprung disease), respectively. A previously reported heterozygous frameshift mutation, c.1024_1040del AGCACGATTCCTTCCAA, (p.S342Pfs*62), on exon 7 of the PAX3 gene was identified in the patient of the fourth family with WS1.
\end{abstract}

Conclusions: An exact description of the mutations responsible for WS provides useful information to explain the molecular cause of clinical features of WS and contributes to better genetic counseling of WS patients and their families.

Keywords: Waardenburg syndrome, MITF, SOX10, PAX3

\footnotetext{
* Correspondence: morovvati@iautmu.ac.ir; morovvati@hotmail.com

${ }^{6}$ Department of Genetics, Faculty of Advanced Sciences and Technology,

Tehran Medical Sciences, Islamic Azad University, Tehran, Iran

Full list of author information is available at the end of the article
}

(c) The Author(s). 2021 Open Access This article is licensed under a Creative Commons Attribution 4.0 International License, which permits use, sharing, adaptation, distribution and reproduction in any medium or format, as long as you give appropriate credit to the original author(s) and the source, provide a link to the Creative Commons licence, and indicate if changes were made. The images or other third party material in this article are included in the article's Creative Commons licence, unless indicated otherwise in a credit line to the material. If material is not included in the article's Creative Commons licence and your intended use is not permitted by statutory regulation or exceeds the permitted use, you will need to obtain permission directly from the copyright holder. To view a copy of this licence, visit http://creativecommons.org/licenses/by/4.0/ The Creative Commons Public Domain Dedication waiver (http://creativecommons.org/publicdomain/zero/1.0/) applies to the data made available in this article, unless otherwise stated in a credit line to the data. 


\section{Background}

Waardenburg syndrome (WS) is an auditory-pigmentary syndrome with an estimated prevalence of approximately $1: 40,000$ in the general population; it is responsible for $2-5 \%$ of congenital deafness in total [1,2]. The disorder is characterized by varying degrees of sensorineural hearing loss and pigmentary abnormalities of the hair, skin, and eye. The severity of such congenital hearing loss varies within/between families, ranging from mild to profound, and is usually non-progressive; it can also be either unilateral or bilateral while bilateral is more common [3-5]. Pigmentary changes of iris in individuals with WS include complete heterochromia iridum that each eye is a different color, partial or segmental heterochromia that segments of blue or brown pigmentation exist in one or both eyes and characteristic brilliant blue in both eyes. Pigmentary disturbances of the hair include white forelock which is observed in at least one-third of individuals with WS type 1 (WS1) or WS type 2 (WS2) and usually involves the forehead but may occur elsewhere; premature greying before age 30 and white hairs can also be evident within eyelashes, eyebrow, or at other sites on the body. On top of that, skin hypopigmentation may be found on the face, trunk, or limbs [4, 6-9].

Depending on the presence or absence of additional symptoms and genetic criteria, WS is divided into four subtypes as in WS1 to WS4. WS1 (OMIM: 193,500) and WS2 (OMIM: 193,510) are the most common types, while WS3 (OMIM: 148,820) and WS4 (OMIM: 277, 580) are not the common types [10-14].

WS1 is an autosomal dominant disorder that is caused by mutations within the gene paired box gene 3 (PAX3) on chromosome $2 \mathrm{q} 36.1$. WS1 is phenotypically similar to WS2, but the presence of dystopia canthorum -a prominent feature of WS1 with a penetrance of $97 \%$ - is specific for WS1. Sensorineural hearing loss is more common in WS2 than WS1 with an incidence rate of $60 \%$ in WS1 to $90 \%$ in WS2 $[5,11,15-17]$.

WS2 is a heterogeneous disorder and is categorized into five subtypes based on the genetic causes. Approximately, $15 \%$ of WS2 cases have mutations in the microphthalmia-associated transcription factor (MITF) gene on chromosome 3p14.1-p12.3, termed WS2A (OMIM: 193,510). Mutations in SRY (sex-determining region Y)-box10 (SOX10) gene on chromosome 22q13.1 are associated with WS2E (OMIM: 611,584) which occurs in about $15 \%$ of WS2 and also mutations in the snail homolog of 2 (SNAI2) gene, mapped to chromosome 8q11.21, result in WS2D (OMIM: 608,890). Two loci embracing WS2B (OMIM: 600,193) and WS2C (OMIM: 606,662) have been localized on chromosome $1 \mathrm{p} 21-\mathrm{p} 13.3$ and $8 \mathrm{p} 23$, respectively $[12,16]$. Also, occasional cases with WS2 are associated with mutations in endothelin-3 (EDN3) and endothelin receptor type B (EDNRB) genes. WS2 is mainly inherited in an autosomal dominant manner, but some cases of WS2 are caused by recessive mutations in the SNAI2 gene. More than half of WS2 patients are not explained at the molecular level $[5,14,18,19]$.

WS3 or Klein-Waardenburg syndrome the severe presentation of WS1- with the autosomal dominant mode of inheritance is caused by heterozygous or homozygous mutations of the PAX3 gene. WS3 is characterized by the presence of musculoskeletal abnormalities of limbs (syndactyly, joint contractures, and muscle hypoplasia) in addition to the common phenotypes of WS1 $[17,20]$.

WS4 or Shah-Waardenburg syndrome is characterized by the association of Hirschsprung disease in addition to clinical features of WS2. Hirschsprung disease (OMIM: $142,623)$ is characterized by the presence of aganglionic megacolon that in turn is caused by the congenital deficiency of neural crest from enteric ganglia. Three disease-causing genes are involved in the pathogenesis of WS4; mutations in EDNRB gene on chromosome $13 q 22.3$ can cause WS4A. Mutations of the EDN3 gene on chromosome 20q13.2-q13.3 have been identified in patients with WS4B. Around $50 \%$ of WS4 patients are justifiable due to SOX10 mutations that are dominantly inherited and are called 'WS4C' while $20-30 \%$ of WS4 patients are due to EDNRB and EDN3 mutations that are inherited in an autosomal recessive pattern. In a nutshell, a minority of WS4 patients cannot be identified molecularly [12, 14, 16, 17, 21, 22].

Though many mutations in MITF, SOX10, and PAX3 genes associated with WS2A, PCWH, and WS1 have been identified respectively in different ethnic groups, there is little to no studies in Iranian populations; thus this study is the first report of a genetically diagnosed case of PCWH in Iranian population. Herein, we aimed to investigate the clinical features and genotypes of four unrelated Iranian patients with WS. In sum, we identified two novel heterozygous mutations in the MITF gene in patients manifesting WS2A, a novel heterozygous mutation in the SOX10 gene in an affected individual with $\mathrm{PCWH}$, and a reported heterozygous mutation in the $P A X 3$ gene in a WS1 patient [5, 23-31].

\section{Case presentation}

Four unrelated Iranian families were referred to Rasad Pathobiology and Genetic Laboratory, Tehran, Iran with the chief complaint of congenital hearing loss. A comprehensive clinical history was gathered and clinical examinations were meticulously performed for each affected individual. Our study included four WS patients and nine additional family members (Figs. 1, 2, 3 and 4A). The clinical features suggested a diagnosis of WS for the probands. 


\section{A)}

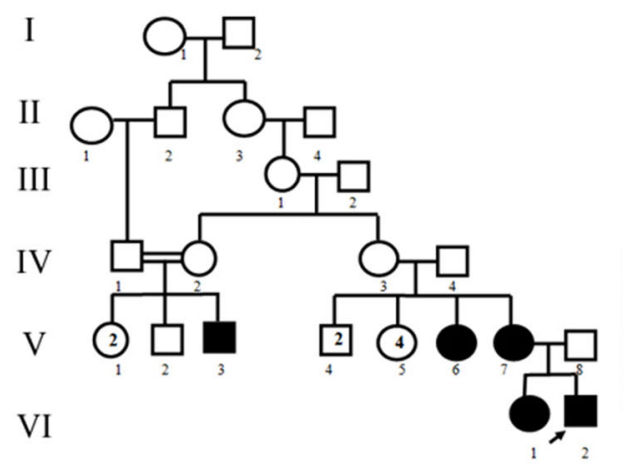

B)
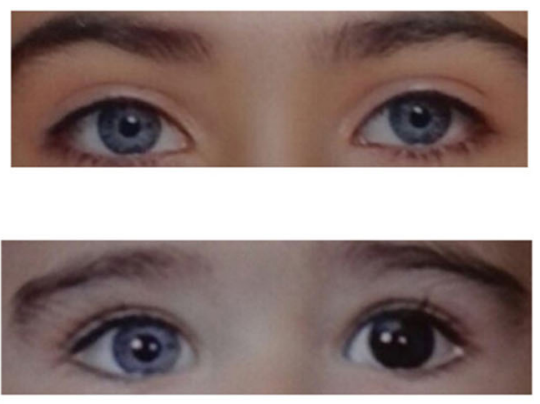

C)

VI-2
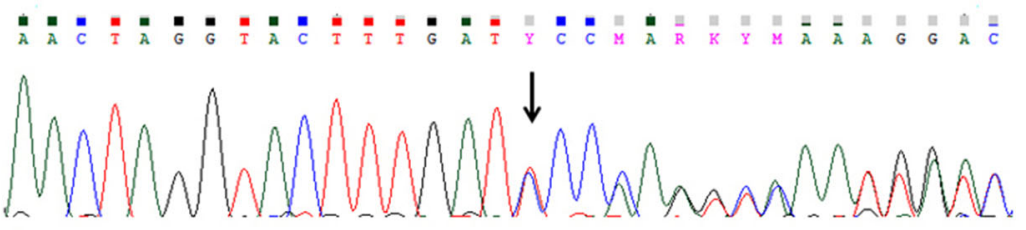

$\mathrm{V}-8$

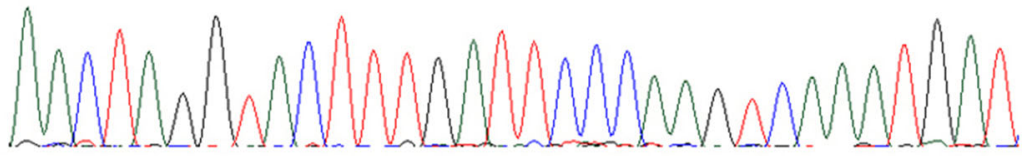

V-7

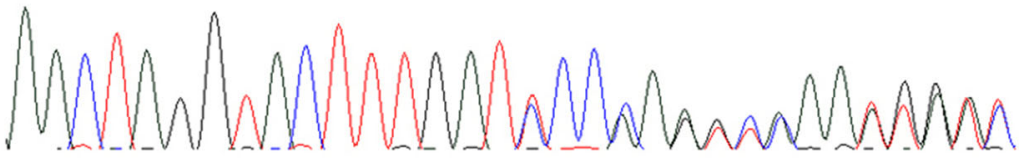

VI-1

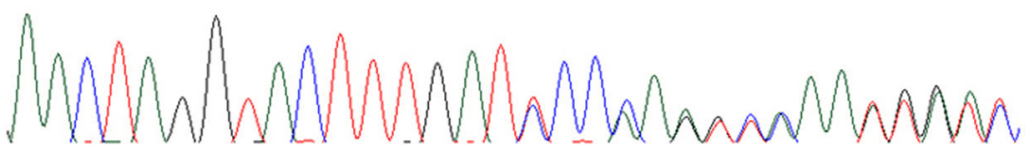

Fig. 1 a Pedigree of family 1 with Waardenburg syndrome type 2A with MITF mutation. The black arrow indicates the proband (VI-2). $\mathbf{b}$ Iris color of Waardenburg syndrome type 2 patients. VI-1, brilliant blue irises. VI-2, heterochromia iridis, and a blue segment in his left iris. c The DNA sequence chromatogram of exon 8 of the MITF gene with the frameshift mutation c.996delT (p.K334Sfs*15) in heterozygous state in proband )VI-2), proband's mother ( $\mathrm{V}-7)$, and proband's sister (VI-1). This mutation was not found in proband's father ( $\mathrm{V}-8)$. The black arrow above the chromatogram sequence shows the c.996delT mutation

Written informed consent was obtained from the patients' parents and all participants included in this study.

Family 1: The proband (VI-2) was the second child of a non-consanguineous marriage and he was a 1-year-old symptomatic boy with congenital hearing loss and heterochromia iridis with the blue segment in his left iris. His older sister (VI-1) was also affected by congenital hearing loss and brilliant blue eyes (Fig. 1B). His mother presented with a white frontal forelock and skin hypopigmentation on her hands and face. His aunt (V-6) and another relative $(\mathrm{V}-3)$ had hearing loss as well. The parents (V-7 and V-8), proband, and his older sister were included in this study.

Family 2: A 1.5-year-old symptomatic girl (III-3) showed a congenital profound hearing loss, brilliant blue eyes, and skin hypopigmentation on the abdominal region at birth but she did not display dystopia canthorum
(Fig. 2B). Her brother (III-2) was healthy, but her maternal grandfather (I-2) and some relatives had brilliant blue eyes. The patient and her healthy parents (II-2 and II-3) were included in this study.

Family 3: The proband (III-2) was an 8-month-old symptomatic male with brilliant blue eyes, congenital profound hearing loss, developmental delay, hypotonia, skin hypopigmentation on her hand and foot, and Hirschsprung disease. Colostomy was performed to remove the non-functional segment of the intestine at age of 2 years. Brain magnetic resonance imaging (MRI) revealed mild atrophy (Fig. 3B). A positive history of hearing loss and polydactyly was detected in the family. The parents were not consanguineous and did not manifest any pertinent clinical features of the disease. The patient and his healthy parents (II-3 and II-4) were included in our study. 
A)

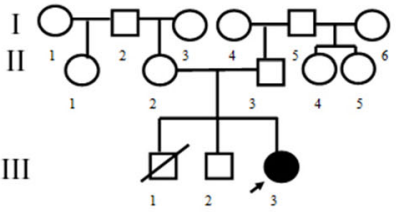

B)

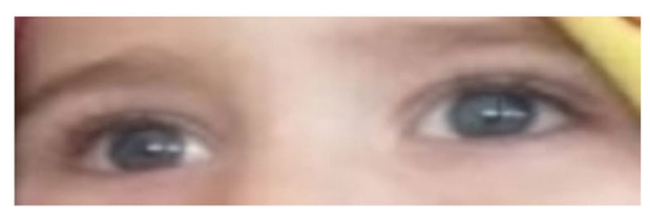

C)

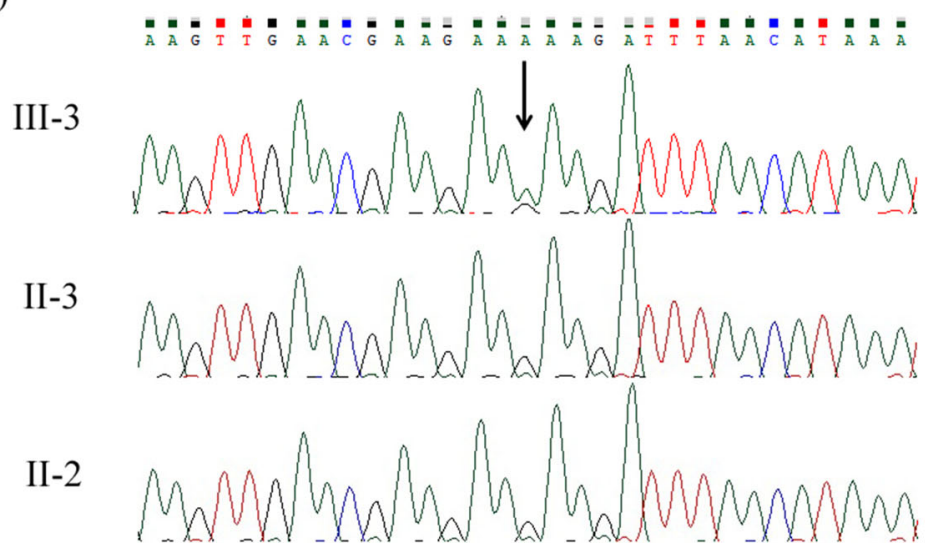

Fig. 2 a Pedigree of family 2 with Waardenburg syndrome type 2A with MITF mutation. The black arrow indicates the proband (III-3). $\mathbf{b}$ Iris color of Waardenburg syndrome type 2 patient. III-3, brilliant blue irises. c The DNA sequence chromatogram of exon 8 of the MITF gene with the missense mutation c.950G > A (p.R317K) in heterozygous state in proband )II-3). This mutation was not detected in proband s father (II-3) and proband s mother (II-2). The black arrow above the chromatogram sequence shows the C.950G > A mutation

Family 4: medical examinations showed that the proband-a 4-year-old symptomatic boy (IV-8)- was suffering from dystopia canthorum, broad nasal root, synophrys, skin hypopigmentation on her hand and abdomen, brilliant blue eyes, and congenital profound hearing loss (Fig. 4B). Five affected individuals (II-6, III4, III-7, III-12, and III-17) exhibited identical phenotypes, but II-6, III-4, III-7, and III-17 had normal hearing. The patients II-6 and III-17did not manifest any pigmentary disturbances of iris. The III-4 and III-7 as the affected individuals presented premature greying before age 30 and heterochromia iridis, respectively. Bilateral congenital hearing loss was evident in IV-4 and IV5; IV-4 had brilliant blue eyes by the way. There was a history of paralysis and cleft lip in the mother's relatives. Two affected patients (IV-8 and III-17) and a healthy individual (III-6) were included in our study.

Whole-exome sequencing was performed on the probands' samples so as to identify the causal genes. To this end, the blood samples were obtained from the proband of each family. Genomic DNA was then extracted from whole blood using standard extraction methods.

Library preparation was performed using the Twist Core Exome kit (TWIST Bioscience, USA) according to the manufacturer's instructions. Sequencing of the libraries was performed by high-throughput paired-end sequencing using the NovaSeq sequencing platform (Illumina Inc., CA, USA) as a service by CeGaT $\mathrm{GmbH}$, Germany.
Alignment to the reference human genome hg19 from UCSC genome browser (University of California, Santa Cruz, USA) was carried out by the Burrows-Wheeler Aligner (BWA) program. Variant calling and filtering were performed using the Genome Analysis Toolkit (GATKv3.4.0). Detected variants were annotated using Ensembl (https://www.ensembl.org), RefSeq (https://www.ncbi.nlm. nih.gov/refseq/), dbSNP (https://www.ncbi.nlm.nih.gov/ snp/), gnomAD (https://www.gnomad.broadinstitute.org), 1000 Genomes (https://www.1000genomes.org) and, OMIM (https://www.omim.org) databases.

To narrow down the derived variants, to begin with, the variants located on genes with no reported human disease were removed from the annotated list of variants. In the next step, variants with alternate to total read ratio of $<0.2$ were removed. Then, the minor allele frequency of $<0.01$ on public databases, e.g. gnomAD and dbSNP, was included as another filter to reduce the number of variants. After applying such filters then variant consequences were used to prioritize remaining variants for further interpretation in terms of pathogenicity based on American College of Medical Genetics and Genomics (ACMG) guideline. Multiple in silico tools including Mutation taster, and CADD were used to evaluate the effect of detected variants on gene or gene products. Evolutionary conservation of genomic position was assessed using Phylop and PhastCons scores. 


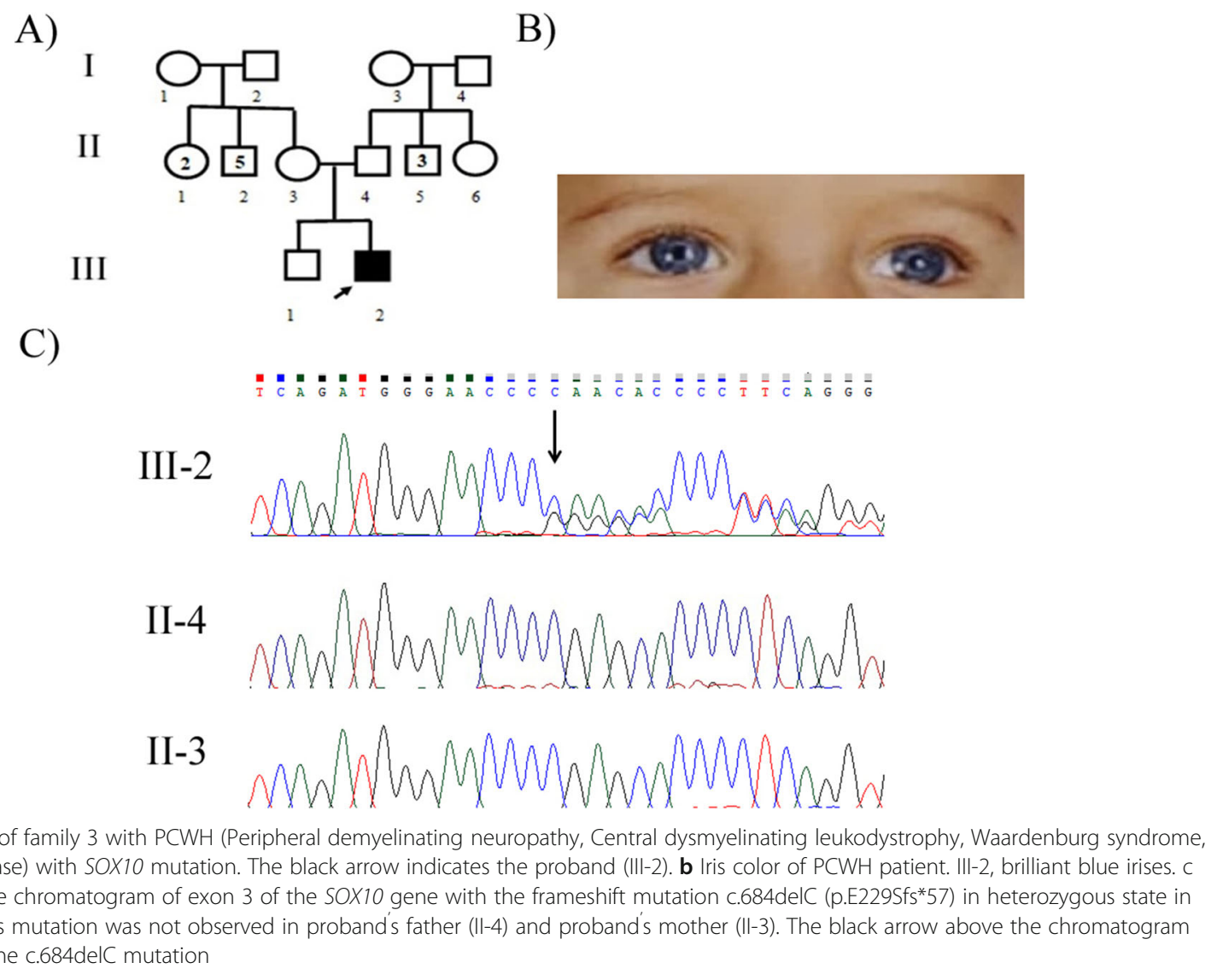

ClinVar (http://www.ncbi.nlm.nih.gov/clinvar/), HGMD (http://www.hgmd.cf.ac.uk/), OMIM, and PubMed were reviewed for previous reports or publications related to pathogenic variants in MITF gene.

The mutations detected by whole-exome sequencing were investigated in patients and all the participants by PCR-Sanger sequencing methods using specific primers. The primers used for amplification has been described in Table 1. The PCR mixture was set in a total volume of $25 \mu \mathrm{l}$. All PCR amplifications were performed using $12.5 \mu \mathrm{l}$ PCR master mix (Yekta Tajhiz Azma, Iran), $8.5 \mu \mathrm{l}$ $\mathrm{H}_{2} \mathrm{O}, 1 \mu \mathrm{l}$ of each primers described in Table 1 , and $2 \mu \mathrm{l}$ extracted DNA. The reaction was started with an initial denaturation at $95^{\circ} \mathrm{C}$ for $5 \mathrm{~min}$, followed by 35 cycles at $95^{\circ} \mathrm{C}$ for $30 \mathrm{~s}$, annealing at various temperature for $45 \mathrm{~s}$ for the different primers, and extension at $72^{\circ} \mathrm{C}$ for $45 \mathrm{~s}$, and a final extension at $72^{\circ} \mathrm{C}$ for $7 \mathrm{~min}$ on a thermal cycler (Veriti, Applied Biosystems, USA). The PCR products were analyzed on $2 \%$ agarose gel and then the products were purified and sequenced on ABI 3500 Genetic analyzer.

In this study, we report four distinct patients of WS in the Iranian population. Using whole-exome sequencing, a frameshift deletion, c.996delT (p.K334Sfs*15), was found in exon 8 of the MITF gene in the first patient
(VI-2). The same mutation was validated in the patient, her affected mother (V-7), and sister (VI-1) in a heterozygous state by PCR and Sanger sequencing methods, but was not detected in his asymptomatic father (V-8) (Fig. $1 \mathrm{C}$ ). Regarding the second patient (III-3), the mutation, c.950G > A (p.R317K), on exon 8 of the MITF was detected by whole-exome sequencing and confirmed by PCR and Sanger sequencing methods in the patient. This mutation was de novo and was not detected in her unaffected parents (II-2 and II-3) with the Sanger sequencing method (Fig. 2 C). In the third patient (III-2), a frameshift deletion, c.684delC (p.E229Sfs*57), in exon 3 of the SOX10 gene was identified in the heterozygous state. However, parental DNA analysis (II-3 and II-4) did not detect any mutation, underscoring that this mutation was de novo(Fig. $3 \mathrm{C}$ ). In the last case (IV-8), a pathogenic frameshift mutation, c.1024_1040del AGCA CGATTCCTTCCAA (p.S342Pfs"62) on exon 7 of the $P A X 3$ gene was detected as a heterozygous mutation. The mutation was co-segregated in all available affected family members (Fig. 4 C) (Table 2).

All detected mutations were analyzed using in silico tools, e.g. MutationTaster and CADD. Available databases were used to review the reported pathogenic variants in MITF, SOX10, and PAX3 genes. All variants were 
A)

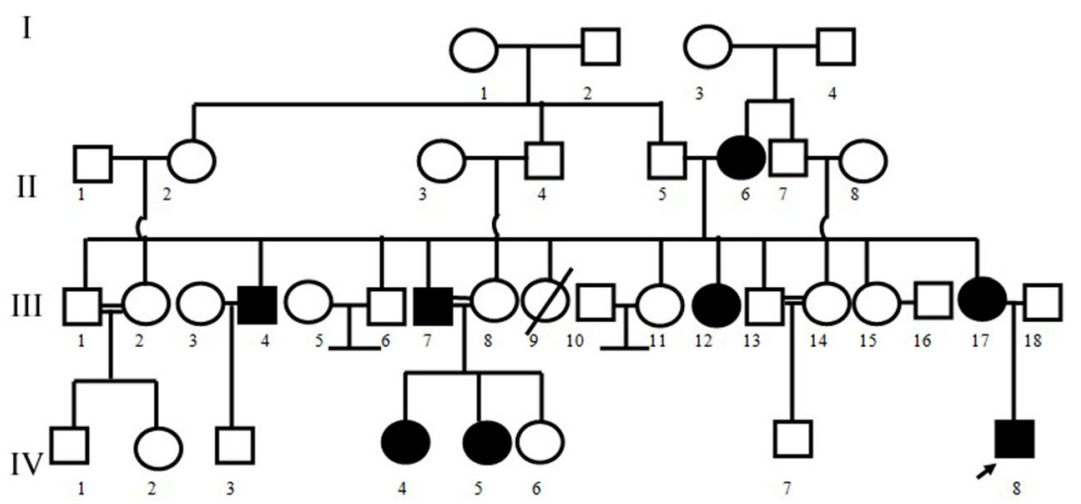

B)

C)
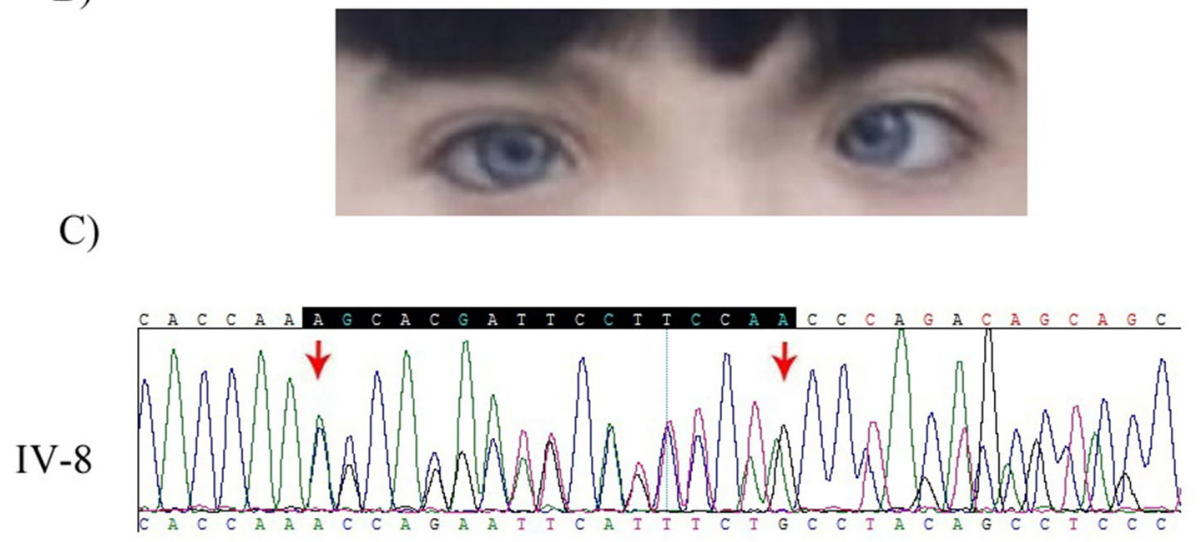

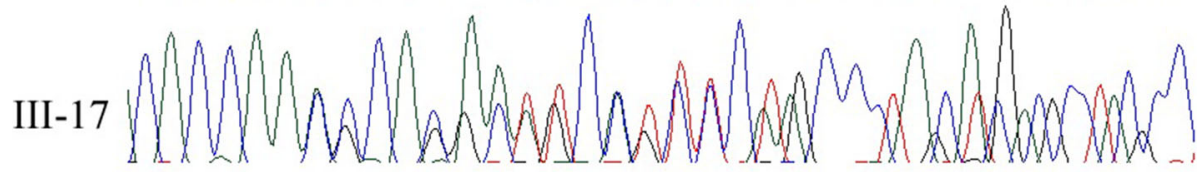

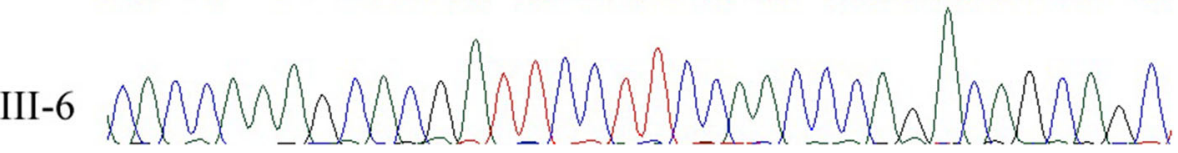

Fig. 4 a Pedigree of family 4 with Waardenburg syndrome type 1 with PAX3 mutation. The black arrow indicates the proband (IV-8). b Iris color of Waardenburg syndrome type 1 patient. IV-8, brilliant blue irises. c The DNA sequence chromatogram of exon 7 of the PAX3 gene with the frameshift mutation c.1024_1040del AGCACGATTCCTTCCAA (p.S342Pfs*62) in heterozygous state in proband (IV-8) and proband's mother (III-17). This mutation was not detected in proband's uncle [mother-side] (III-6). The black arrow above the chromatogram sequence shows the c.1024_1040del AGCACGATTCCTTCCAA mutation

Table 1 PCR Primers Sequences

\begin{tabular}{|c|c|c|c|}
\hline Gene exon & $\begin{array}{l}\text { Forward primer } \\
\text { sequence }\left(5^{\prime}-3^{\prime}\right)\end{array}$ & $\begin{array}{l}\text { Reverse primer } \\
\text { sequence }\left(5^{\prime}-3^{\prime}\right)\end{array}$ & $\begin{array}{l}\text { Product length } \\
\text { (bp) }\end{array}$ \\
\hline MITF-exon8 & AGAATTTGGGCTTTCACCAG & CTTGATGAGACTAACCAAAAGAAAG & 590 \\
\hline sox10-exon3 & CCAGCCCATGAA AGATTTGG & TGCCCCAGCCACCTCTC & 1,090 \\
\hline PAX3-exon7 & GGTGGCTGATGAACTITTGC & AGAAACACGGGACTGACCTG & 313 \\
\hline
\end{tabular}


Table 2 Identified variants in this study

\begin{tabular}{|c|c|c|c|c|c|c|c|}
\hline $\begin{array}{l}\text { Gene/ Transcript } \\
\text { (RefSeq) }\end{array}$ & $\begin{array}{l}\text { Variant } \\
\text { Location } \\
\end{array}$ & Variant & $\begin{array}{l}\text { Chromosome } \\
\text { Position (GRCh37) }\end{array}$ & Zygosity $^{a}$ & $\begin{array}{l}\text { OMIM } \\
\text { number }\end{array}$ & $\begin{array}{l}\text { Inheritane } \\
\text { Pattern }^{c}\end{array}$ & $\begin{array}{l}\text { Variant } \\
\text { Classification }^{\mathrm{d}}\end{array}$ \\
\hline $\begin{array}{l}\text { MITF NM_- } \\
198159.3\end{array}$ & Exon 8 & c.996delT p.K334Sfs*15 & $\begin{array}{l}\text { Chr3: } \\
70,005,662\end{array}$ & Het & 193,510 & $A D$ & Pathogenic \\
\hline $\begin{array}{l}\text { MITF NM_- } \\
198159.3\end{array}$ & Exon 8 & c.950G > A p.R317K & $\begin{array}{l}\text { Chr3: } \\
70,005,618\end{array}$ & Het & 193,510 & $A D$ & $\begin{array}{l}\text { Likely } \\
\text { pathogenic }\end{array}$ \\
\hline $\begin{array}{l}\text { SOX10 NM_ } \\
006941.4\end{array}$ & Exon 3 & c.684delC p.E229Sfs*57 & Chr22: 38,373,887 & Het & 609,136 & $A D$ & pathogenic \\
\hline $\begin{array}{l}\text { PAX3 NM_ } \\
181459.4\end{array}$ & Exon 7 & $\begin{array}{l}\text { c.1024_- } \\
\text { 1040delAGCACGATTCCTTCCAA } \\
\text { p.S342Pfs*62 }\end{array}$ & Chr2: $223,084,992$ & Het & 193,500 & $A D$ & Pathogenic \\
\hline
\end{tabular}

${ }^{\mathrm{a}}$ Het Heterozygous, ${ }^{\mathrm{b}} \mathrm{OMIM}$ number Five-digit number assigned to each phenotype in Online Mendelian Inheritance in Man (OMIM) database, ${ }^{\mathrm{c}} A D$ Autosomal dominant. ${ }^{d}$ Based on American College of Medical Genetics and Genomics (ACMG) standards and guidelines for the interpretation of sequence variants, 2015 $*$ = termination codon

classified as the "pathogenic variants" based on ACMG guidelines.

\section{Discussion and conclusions}

In this report, we describe the clinical features of four genotyped unrelated cases with frameshift deletions in the MITF, SOX10, and PAX3 genes and a missense mutation in the basic domain of the MITF gene.

WS2A -a subset of WS2- is an autosomal dominant disorder characterized by variable degrees of sensorineural hearing loss and pigmentary disturbances of skin, hair, and eyes. Heterozygous mutations in the MITF gene cause WS2A and lack of normal melanocytes in the affected organs, e.g. eye, skin, and the cochlea [3234]. In the patient of family 1 , a heterozygous novel mutation, c.996delT (p.K334Sfs"15), was identified in exon 8 of the MITF. This mutation was a single-base deletion of a $\mathrm{T}$ at base 996 within the loop of the MITF protein, resulting in a frameshift and premature stop codon at 15 codons downstream from the deletion point. Therefore, it is truncated at the second helix portion lacking twelve residues of the second helix and the complete leucine zipper region. This mutation results in a truncated MITF protein with 348 amino acids instead of the wild type length of 520 amino acids. Therefore, about $33 \%$ of the protein length has been deleted $[35,36]$. No dystopia canthorum, musculoskeletal abnormalities, or Hirschsprung disease was observed in affected members of this family and the pedigree chart showed autosomal dominant inheritance. Based on Waardenburg Consortium, clinical manifestations and the detected variant in the MITF gene in our patients, diagnosis of WS2A was confirmed [6]. Multiple lines of in silico computational analysis (e.g. CADD, Mutation Taster, etc.) supported the deleterious effect of the variant. The bHLHZip structure of MITF has an important role in its function and an important role of the HLHZip is in dimerization. The mutation found in MITF seems that disrupted the bHLHZip domain and impaired the function of MITF protein and it is thought to have defects in homo- or heterodimerization and DNA binding. As a result, this can affect the expression of its target genes which leads to the absence of normal melanocytes in the skin, eye, and the cochlea [34,37]. Previous studies indicated that the stop mutation p.R255" and p.R259* in the MITF exon 8 in patients with WS2A resulted in truncated MITF proteins in the second helix domain, thus, lost their DNA binding ability and homo- or heterodimerization activity and also p.R259* failed to activate expression of the TYR, TYRP1, and DCT genes. Also, Yan et al. in 2011 reported a heterozygous mutation c.742 743delAAinsT;746_747delCA in exon 8 of the MITF gene in a large Chinese family with WS2A that this mutation resulted in a premature termination codon within the second helix of MITF protein. Therefore, a nonfunctional MITF protein was produced due to loss of the bHLHZip structural domain and was led to haploinsufficiency [12, 35, 38, 39].

The microphthalmia (MiT) family of transcription factors includes MITF, TFEB, TFEC and TFE3 which share a common basic-helix-loop-helix-leucine zipper (bHLHZip) dimerization motif and a transactivation domain (TAD). Nine different MITF isoforms have been produced from a single MITF gene and all isoforms have exons 2-9 in common [36]. MITF is expressed in melanocytes and contains a basic domain for DNA binding and an HLHZip domain for homo- or heterodimerization with either TFEB, TFEC, or TFE3, which recognizes specific DNA sequences of pigmentation genes, including tyrosinase (TYR), tyrosinase-related protein 1 (TRP1) and DCT/ TRP2 that are responsible for melanogenesis and regulates the expression of these genes; therefore, it has an important role in melanocytes development and differentiation. MITF isoform A is composed of 9 exons, which consists of 520 amino acid residues. This transcription factor is expressed in many cell types including melanocytes and retinal pigment epithelium (RPE) cells [12, 35, $37,40-42]$. 
A previous study demonstrated that mutations of Ser298 in MITF-M (Ser399 in MITF-A), which is located downstream of bHLHZip, has an important role in the transcriptional function of MITF. It has been shown that phosphorylation of Ser298 by Glycogen synthase kinase 3 (GSK3) enhances the ability of MITF to bind the DNA in vitro. Consequently, the phosphorylation of MITF by GSK3 $\beta$ in this truncated protein is abolished impairs the function of MITF that can be a potential cause of WS2. Also, serine-rich and threonine-rich regions of MITF are important for its function. Thus, transactivation assays revealed that truncation of the last 95 amino acids (including threonine- and serine-rich regions) decreased the transcriptional activity of MITF (MITF-M[1-324], MITF-A[1-425]. Transactivation assays and DNA-binding assays revealed that deletion of (MITF-M[294-324], MITF-A[395-425]) which is downstream of the bHLHZip structure, decreased the transactivation activity and ability of MITF to bind to the Ebox, respectively. As a result, the truncated mutation -p.(K334Sfs"15)- does not have the last 95 amino acids and also the amino acids from 395 to 425 that can affect its transcriptional activity, transactivation capacity, and DNA binding ability [35, 37]. The mutation described in this study results in a premature translational termination codon in the last translated exon, thus, it is predicted that induce escaping the mutant transcript from the nonsense-mediated mRNA decay (NMD) pathway $[11,19]$. Nobukuni et al., showed that the MITF proteins with truncated mutations within the bHLHZip region lost their ability to dimerize with wild-type MITF proteins, but they did not have any interfering with DNA binding activity of wild-type MITF protein, therefore, the clinical characteristics of patients with WS2A are caused by loss of function mutations resulting in haploinsufficiency of the MITF protein. Haploinsufficiency appears to be a major mechanism in deafness and pigmentary abnormalities of WS2 patients [34, 37]. Different kinds of mutations have been reported in MITF such as missense, nonsense, and splicing mutations [43].

A missense mutation, c.950G > A (p.R317K), in the MITF-A, corresponding to p.Arg216Lys in the MITF-M (GenBank: NM_000248.1), was identified in exon 8 of the MITF in the second patient with WS2A and resulted in an amino acid arginine being replaced by lysine at residue 317. This novel and non-truncating variant is located at the basic domain of the MITF protein [35]. Therefore, it would be expected to impair the DNA binding ability of MITF protein, and it is thought to be responsible for the disease. The proband's parents did not carry the same mutation indicating that the mutation occurred de novo. Tietz syndrome (TS; OMIM: 103,500) is a more severe form of WS2A and it is characterized by profound congenital deafness and generalized hypopigmentation of skin, hair, and eye that is transmitted in an autosomal dominant manner with heterozygous mutations within the MITF gene, while WS2A is differentiated from Tietz syndrome by patchy depigmentation abnormalities of the skin, hair, and irides [32, 33]. Our patients with MITF mutations had a less severe phenotype without generalized hypopigmentation of skin, hair, and eye. A TS-causing mutation of p.Arg216Ser and a de novo missense mutation p.Arg216Lys, which are located at the basic domain of the MITF protein, have been previously reported. Although these two mutations are located at the same amino acid position, Arg216Ser caused a more severe phenotype than a missense mutation p.Arg216Lys, thus the type of amino acid in the location of the mutation in the protein can be an important factor in the determination of TS and WS2A phenotype. Also, WS2A-associated p.Arg216Lys was demonstrated to lack DNA binding and activate expression from the TYR, TYRP1, and DCT promoters. Arg216 is a conserved residue in MITF proteins among different species [32, 35, 38]. De novo missense mutations p.Glu213Asp, p.Arg216Lys, p.Arg217Gly, and p.Arg217Ile, in patients with hearing loss and blue irides, located at the basic domain of the MITF protein have been previously reported associated with WS2A and failed to bind DNA and activate expression of the TYR, TYRP1, and DCT genes $[12,23,38,44]$. Also, heterozygous mutations p.Lys307Asn, p.Leu312fs*11, p.A315Arg (c.944_946delGAA), p.Arg318Gly and p.Arg318del within the basic domain of MITF-A has been reported to cause WS2A [8, 35, 45]. According to the Leiden Open Variation Database (http://grenada.lumc.nl/LOVD2/WS/), the majority of MITF mutations are located in exons 7, 8, and 9 that encode the bHLH-zip domain $[3,8]$. This variant is predicted to be damaging by in silico analysis using SIFT.

Regarding patient 3, a SOX10 heterozygous mutation, c.684delC (p.Glu229Serfs*57), was identified. This SOX10 frameshift mutation is proximal to the 3'-end of exon 3 and is localized at the intervening sequence of the HMG domain and $\mathrm{K} 2$ region which shifts the reading frame after codon 229, resulting in a premature termination codon within the $\mathrm{K} 2$ conserved region of the SOX10 protein. Therefore, the truncated SOX10 protein has 285 amino acids instead of the wild type length of 466 amino acids [22]. SOX10 is a member of the SOX family of transcription factors involved in the development of the enteric nervous system (ENS), melanocytes, glial cells, and intestinal ganglia cells. It has $4 / 5$ exons with three coding exons which encode a protein of 466 amino acids. SOX10 possesses a dimerization domain (AA61-101), a DNAbinding high-mobility group (HMG) domain (101-180), a conserved domain in the center of the protein (233-306), and a transactivation domain $(400-466)$ at the Cterminus. Mutations in SOX10 are associated with WS2E and WS4C (OMIM: 613,266), while mutations in SOX10 
that are responsible for WS4 with neurological features referred to as PCWH (OMIM: 609,136) which patients may suffer from peripheral neuropathy, mental retardation, cerebellar ataxia, neonatal hypotonia and spasticity $[5,14$, 46-48]. PCWH is mostly caused by truncating mutations in the last coding exon of SOX10 or less than 50-55 nucleotides upstream of the last coding exon, induce escaping the mutant mRNAs from the NMD pathway. Thus, the synthesized mutant protein act as a dominantnegative protein that interferes with the activity of the wild-type SOX10, resulting in neurological symptoms in WS. The previous report showed that a WS patient with a truncating mutation of c.652G > T, p.G218* in exon 3 of the SOX10 had neurological symptoms [47]. SOX10 indeed interacts with PAX3 and regulates the MITF expression, so it can be hypothesized that SOX10 or PAX3 dysfunction impairs MITF expression. Also, in vitro studies showed that the S251X mutation within the conserved domain of SOX10 abolishes The SOX10-dependent activation as well as synergistic activation of the MITF promoter with PAX3. The conserved region in SOX10 has a crucial role in mediating the synergy between SOX10 and PAX3; hence, axiomatically, any truncating mutations in this region can impair the ability of SOX10 to interact with PAX3 $[5,10]$. Consistent with the previous study it is predicted that the truncating mutation c.684delC (p.Glu229SerfsTer57) identified in this study loses its ability to activate MITF promoter and its interaction with PAX3. This mutation results in a premature translational termination codon in the last coding exon of SOX10 that escapes the NMD pathway and act as a dominantnegative protein that interferes with the activity of the wild-type SOX10. This result underscored that the clinical manifestations of this patient were in line with this mutation [22]. The SOX10 mutations associated with the classical form of WS4 and PCWH are present heterozygously and most often occur de novo [5]. The analysis of the unaffected parents'DNA by PCR-Sanger sequencing revealed the de novo occurrence of the mutation. Multiple lines of in silico computational analysis (CADD, Mutation Taster, etc.) supported the deleterious effect of the variant. The majority of the SOX10 mutations are embracing the truncating mutations such as nonsense and frameshift. The other types of mutations comprise splice mutations, inframe insertions, substitution, and partial or full gene deletions [5, 49]. Previous studies have reported some PCWH-related mutations including p.Gln234X, p.Gln250X, p.Ser251X, p.Gly266AlafsX20, and p.His283LeufsX11 in SOX10 conserved domain [28].

In the present study, the WES revealed a heterozygous mutation c.1024_1040del AGCACGATT CCTTCCAA (p.Ser342ProfsX62) in exon 7 of the PAX3 gene in the fourth patient with dystopia canthorum, a typical characteristic of WS1, which has been reported previously. This 17-base deletion occurred within the TAD of the PAX3 gene and altered the reading-frame followed by premature translational of a stop codon in TAD. This truncating mutation can lead to a truncated PAX3 protein with 403 amino acids instead of the wild type length of 505 amino acids, it has functional paired domain and homeodomain but a loss of a part of TAD would be expected to affect its normal function. PAX3 encodes a member of the paired box of transcription factors that is involved in the development of the central nervous system, skeletal muscle, and melanocytes. There are eight human PAX3 isoforms, among which, PAX3e is the longest isoform consists of 10 exons and encodes a paired domain (PD) by exons $2-4$, a homeodomain (HD) by exons 5 and 6 , and a Ser-Thr-Pro-rich $C$ terminus transactivation domain (TA) by exons $6-8$. The PD and HD are DNA binding domains, while the TA domain mediates transcriptional regulation. The translated regions encode a 505 -amino acid protein $[5,19,48,50,51]$. A previous functional study has demonstrated that the truncating mutation 1185 insTGA which removes a part of the transactivation domain of PAX3 reduced its effect on MITF promoter activation, and also its synergistic effect with SOX10 was completely lost. As a result, it would be expected that the c.1024_1040del AGCACG ATTCCTTCCAA mutation found in this study causes losing its ability to activate MITF promoter and its interaction with SOX10 [10]. WS1 is caused by loss of function mutations in the $P A X 3$ gene leading to haploinsufficiency [34, 52].

All WS clinical features show variable expressivity among the affected individuals [4, 11, 39]. Therefore, there was considerable variability in phenotypes of members of this family. We found that 5 out of 8 $(62.5 \%)$ affected members of family four had iris pigmentary disturbances including brilliant blue eyes and complete heterochromia iridis. Four affected individuals $(50 \%)$ had hearing loss while other patients had normal hearing. Dystopia canthorum, broad nasal root, synophrys, and skin hypopigmentation were observed in 6 patients $(75 \%)$ of this family. No musculoskeletal abnormalities were detected in each patient of the family. The father (III-18) was healthy and there was not any phenotype of the disease in anybody of the next of the kins. Thus, analysis of his affected mother's DNA by PCR-Sanger sequencing revealed that she was the carrier of the same heterozygous mutation but failed to demonstrate the mutation in his healthy uncle (III-6). According to Waardenburg Consortium, clinical features of our patient and the genetic mutation in the PAX3, he was classified into the WS1 group [7]; the pedigree analysis showed that the variant was segregated with WS phenotypes as an autosomal dominant trait in this family. Most patients of WS1 manifest heterozygous mutations in the $P A X 3$ gene [53]. Different $P A X 3$ mutations including missense, nonsense, frameshifts, 
small in-frame indels, and splice alterations have been reported in WS1 patients; for instance, some WS1related mutations have been reported that in turn include p.Gly292ArgfsX118, p.Phe294ValfsX116, p.Pro318HisfsX63, and p.Leu396X in the PAX3 transactivation domain [5, 31].

Based on the ACMG criteria [54], the c.996delT variants of MITF, c.684delC of SOX10, and c.1024_1040del AGCACGATTCCTTCCAA of $P A X 3$ were considered to be pathogenic and c.950G > A of MITF is likely pathogenic. The three reported variants c.996delT, c.950G > $\mathrm{A}$, and c.684delC in this study are absent in population databases such as Exome Aggregation Consortium (ExAC) (http://exac.broadinstitute.org/), 1000 Genomes (http://www.1000genomes.org/), dbSNP (http://www. ncbi.nlm.nih.gov/snp/) and our local database.

In conclusion, we identified three novel as well as a previously reported mutation in MITF, SOX10, and $P A X 3$ gene in four unrelated Iranian families. Three mutations reported in this study including c.996delT (p.K334Sfs"15) in exon 8 of the MITF, c.684delC (p.Glu229Serfs*57) in exon 3 of the SOX10 and c.1024 1040del AGCACGATTCCTTCCAA (p.Ser342Profs"62) in exon 7 of the $P A X 3$ gene are frameshift and truncating mutations which ultimately results in early termination, leading to the formation of a truncated protein that can affect their normal function and a missense mutation reported in this study impairs DNA binding ability of MITF protein. Functional analysis is required to explore the exact mechanisms of pathogenesis of these mutations. An exact description of the mutations responsible for WS provides useful information to explain the molecular cause of clinical features of WS and contribute to better genetic counseling of WS patients and their families. Also, our study expands the mutation spectrum of MITF, SOX10, and PAX3 genes.

\section{Abbreviations}

WS: Waardenburg syndrome; PAX3: Paired box gene 3;

MITF: Microphthalmia-associated transcription factor; SOX10: SRY (sexdetermining region Y)-box10; SNAI2: Snail homolog of 2; EDNRB: Endothelin receptor type B; EDN3: Endothelin-3; WES: Whole-exome sequencing; MRI: Magnetic resonance imaging; MiT: Microphthalmia; bHLHZip: Basic-helixloop-helix-leucine zipper; TAD: Transactivation domain; TYR: Tyrosinase; TRP1: Tyrosinase-related protein 1; RPE: Retinal pigment epithelium; GSK3: Glycogen synthase kinase 3; NMD: Nonsense-mediated mRNA decay; TS: Tietz syndrome; ENS: The enteric nervous system; HMG: High-mobility group; PCWH: Peripheral demyelinating neuropathy, Central dysmyelinating leukodystrophy, Waardenburg syndrome, Hirschsprung disease; PD: Paired domain; HD: Homeodomain

\section{Acknowledgements}

The authors sincerely thank the patients and their family members for their participation in this study and the staff of the Rasad Pathobiology and Genetic Laboratory, Tehran, Iran. We would like to thank Mr. Ehsan Razmara, Tarbiat Modares University, Tehran, Iran for his efficient and excellent language editing of our manuscript.

\section{Authors' contributions}

$\mathrm{SM}$ is responsible for the design of this study, acquisition, analysis, and interpretation of data for the work. SZ drafted the work; SM, SR, MHD, and AA revised the draft critically for important intellectual content; SM, SZ, and SR provided approval for publication of the content; SZ, SR, SM, and MHD collected the detailed information; MK and ZJ had cooperation in the revision of the manuscript; all authors read and approved the final manuscript.

\section{Funding}

This study was supported by Rasad Pathobiology and Genetic Laboratory, Tehran, Iran that had an important role in the collection and analysis of data but it did not have any role in the design of the study, interpretation of data and writing the manuscript.

\section{Availability of data and materials}

The datasets used and/or analyzed during the current study are available from the corresponding author on reasonable request.

\section{Ethics approval and consent to participate}

This study was approved by the ethical committees of Rasad Pathobiology and Genetic Laboratory, Tehran, Iran. Written informed consent to participate was obtained from the patients' parents and all participants (family members who underwent genetics testing etc.) of this study.

\section{Consent for publication}

Written informed consent was obtained from the patients' parents and all participants (family members who underwent genetics testing) for publication of this case report and any accompanying images.

\section{Competing interests}

There is no conflict of interest in this article.

\section{Author details}

${ }^{1}$ Department of Biology, School of Basic Sciences, Science and Research Branch, Islamic Azad University, Tehran, Iran. ${ }^{2}$ Tehran Medical Sciences, Islamic Azad University, Tehran, Iran. ${ }^{3}$ Faculty of Medicine, Hormozgan University of Medical Sciences, Hormozgan, Iran. ${ }^{4}$ Department of Medical Genetics, Faculty of Medicine, Tehran University of Medical Sciences, Tehran, Iran. ${ }^{5}$ School of Advanced Sciences and Technology, Islamic Azad University-Tehran Medical Sciences, Tehran, Iran. ${ }^{6}$ Department of Genetics, Faculty of Advanced Sciences and Technology, Tehran Medical Sciences, Islamic Azad University, Tehran, Iran.

Received: 10 September 2020 Accepted: 24 January 2021 Published online: 08 February 2021

\section{References}

1. Chen Y, Yang F, Zheng H, Zhou J, Zhu G, Hu P, et al. Clinical and genetic investigation of families with type II Waardenburg syndrome. Mol Med Rep. 2016;13(3):1983-8.

2. Li W, Mei L, Chen H, Cai X, Liu Y, Men M, et al. New genotypes and phenotypes in patients with 3 subtypes of Waardenburg syndrome identified by diagnostic next-generation sequencing. Neural Plast. 2019; 2019(endothelin3):7143458

3. Sun J, Hao Z, Luo H, He C, Mei L, Liu Y, et al. Functional analysis of a nonstop mutation in MITF gene identified in a patient with Waardenburg syndrome type 2. J Hum Genet. 2017;62(7):703-9. https://doi.org/10.1038/ jhg.2017.30.

4. Haj Kassem L, Ahmado MF, Sheikh Alganameh M. A rare case of seven siblings with Waardenburg syndrome: A case report. J Med Case Rep. 2018; 12(1):1-5

5. Pingault V, Ente D, Dastot-Le Moal F, Goossens M, Marlin S, Bondurand N Review and update of mutations causing Waardenburg syndrome. Hum Mutat. 2010;31(4):391-406.

6. Liu XZ, Newton VE, Read AP. Waardenburg syndrome type II: Phenotypic findings and diagnostic criteria. Am J Med Genet. 1995;55(1):95-100.

7. Farrer LA, Grundfast KM, Amos J, Arnos KS, Asher JH, Beighton P, et al. Waardenburg syndrome (WS) type I is caused by defects at multiple loci, one of which is near ALPP on chromosome 2: First report of the WS consortium. Am J Hum Genet. 1992;50(5):902-13. 
8. Shi Y, Li X, Ju D, Li Y, Zhang X, Zhang Y. A novel mutation of the MITF gene in a family with Waardenburg syndrome type 2: A case report. Exp Ther Med. 2016;11(4):1516-8.

9. Vichare N, Bhargava N. Waardenburg syndrome: A rare case with bilateral congenital cataract: An unusual entity. Med J Armed Forces India. 2013; 69(2):172-4.

10. Bondurand N, Pingault V, Goerich DE, Lemort N, Sock E, Le Caignec C, et al. Interaction among SOX10, PAX3 and MITF, three genes altered in Waardenburg syndrome. Hum Mol Genet. 2000;9(13):1907-17.

11. Morell R, Spritz RA, Ho L, Pierpont J, Guo W, Friedman TB, et al. Apparent digenic inheritance of Waardenburg syndrome type 2 (WS2) and autosomal recessive ocular albinism (AROA). Hum Mol Genet. 1997;6(5):659-64.

12. Yang S, Dai P, Liu X, Kang D, Zhang X, Yang W, et al. Genetic and phenotypic heterogeneity in Chinese patients with Waardenburg syndrome type II. PLoS One. 2013;8(10):e77149.

13. Khan TA, Safdar CA, Zameer S, Khushdil A. Waardenburg-Shah syndrome (WS type IV): a rare case from Pakistan. Perioper Med. 2020;9(1):1-3.

14. Song J, Feng Y, Acke FR, Coucke P, Vleminckx K, Dhooge IJ. Hearing loss in Waardenburg syndrome: A systematic review. Clin Genet. 2016;89(4):416-25.

15. Yoshida Y, Doi R, Adachi K, Nanba E, Kodani I, Ryoke K. A novel PAX3 mutation in a Japanese boy with Waardenburg syndrome type 1. Hum Genome Var. 2016;3(January):2015-7.

16. Kapoor S, Bindu PS, Taly AB, Sinha S, Gayathri N, Vasantha Rani S, et al. Genetic analysis of an Indian family with members affected with Waardenburg syndrome and Duchenne muscular dystrophy. Mol Vis. 2012; 18(July):2022-32

17. Nusrat M, Tariq MA, Aslam S, Zil-E-Ali A, Shahid M, Mahmood S. A case of Waardenburg-Shah syndrome type 4 presenting with bilateral homochromatic blue Irises from Pakistan. Cureus. 2018;10(8):e3143.

18. Sánchez-Martín M, Rodríguez-García A, Pérez-Losada J, Sagrera A, Read AP, Sánchez-García I. SLUG (SNA12) deletions in patients with Waardenburg disease. Hum Mol Genet. 2002;11(25):3231-6.

19. Shelby M. Waardenburg syndrome expression and penetrance. J Rare Dis Res Treat. 2017;2(6):31-40.

20. Read AP, Newton VE. Waardenburg syndrome. J Med Genet. 1997;34(8):65665. https://doi.org/10.1136/jmg.31.10.744.

21. Price ER, Fisher DE. Sensorineural deafness and pigmentation genes: Melanocytes and the Mitf transcriptional network. Neuron. 2001;30(1):15-8.

22. Fernández RM, Núñez-Ramos R, Enguix-Riego MV, Román-Rodríguez FJ, Galán-Gómez E, Blesa-Sánchez E, et al. Waardenburg syndrome type 4: Report of two new cases caused by SOX10 mutations in Spain. Am J Med Genet Part A. 2014;164(2):542-7.

23. Wildhardt G, Zirn B, Graul-Neumann LM, Wechtenbruch J, Suckfüll M, Buske A, et al. Spectrum of novel mutations found in waardenburg syndrome types 1 and 2: Implications for molecular genetic diagnostics. BMJ Open. 2013;3(3):e001917.

24. Chen H, Jiang L, Xie Z, Mei L, He C, Hu Z, et al. Novel mutations of PAX3, MITF, and SOX10 genes in Chinese patients with type I or type II Waardenburg syndrome. Biochem Biophys Res Commun. 2010;397(1):70-4. https://doi.org/10.1016/j.bbrc.2010.05.066.

25. Tassabehji M, Newton VE, Liu XZ, Brady A, Donnai D, Krajewska-walasek M, et al. The mutational spectrum in waardenburg syndrome. Hum Mol Genet. 1995;4(11):2131-7.

26. Jalilian N, Tabatabaiefar MA, Bahrami T, Karbasi G, Bahramian MH, Salimpoor $A$, et al. A novel pathogenic variant in the MITF gene segregating with a unique spectrum of ocular findings in an extended Iranian Waardenburg syndrome kindred. Mol Syndromol. 2017;8(4):195-200.

27. Jalilian N, Tabatabaiefar MA, Yazdanpanah M, Darabi E, Bahrami T, Zekri A, et al. A comprehensive genetic and clinical evaluation of Waardenburg syndrome type II in a set of Iranian patients. Int J Mol Cell Med. 2018;7(1): $17-23$.

28. Harris ML, Baxter LL, Loftus SK, Pavan WJ. Sox proteins in melanocyte development and melanoma. Pigment Cell Melanoma Res. 2010;23(4):496-513.

29. Baldwin CT, Hoth CF, Macina RA, Milunsky A. Mutations in PAX3 that cause Waardenburg syndrome type I: Ten new mutations and review of the literature. Am J Med Genet. 1995;58(2):115-22.

30. Jalilian N, Tabatabaiefar MA, Farhadi M, Bahrami T, Noori-Daloii MR. A novel mutation in the PAX3 gene causes Waardenburg syndrome type I in an Iranian family. Int J Pediatr Otorhinolaryngol. 2015;79(10):1736-40. https:// doi.org/10.1016/j.jpporl.2015.07.039.
31. Jalilian N, Tabatabaiefar MA, Farhadi M, Bahrami T, Emamdjomeh H, NooriDaloii MR. Molecular and clinical characterization of Waardenburg syndrome type I in an Iranian cohort with two novel PAX3 mutations. Gene. 2015; 574(2):302-7. https://doi.org/10.1016/.gene.2015.08.023.

32. Cortés-González V, Zenteno JC, Guzmán-Sánchez M, Giordano-Herrera V, Guadarrama-Vallejo D, Ruíz-Quintero N, et al. Tietz/Waardenburg type 2A syndrome associated with posterior microphthalmos in two unrelated patients with novel MITF gene mutations. Am J Med Genet Part A. 2016; 170(12):3294-7.

33. Steingrímsson E, Copeland NG, Jenkins NA. Melanocytes and the Microphthalmia transcription factor network. Annu Rev Genet. 2004; 38(February):365-411.

34. Nobukuni Y, Watanabe A, Takeda K, Skarka H, Tachibana M. Analyses of loss-offunction mutations of the MITF gene suggest that haploinsufficiency is a cause of Waardenburg syndrome type 2A. Am J Hum Genet. 1996;59(1):76-83.

35. Amae S, Fuse N, Yasumoto Kl, Sato S, Yajima I, Yamamoto H, et al. Identification of a novel isoform of microphthalmia-associated transcription factor that is enriched in retinal pigment epithelium. Biochem Biophys Res Commun. 1998:247(3):710-5.

36. Hartman ML, Czyz M. MITF in melanoma: Mechanisms behind its expression and activity. Cell Mol Life Sci. 2015;72(7):1249-60.

37. Takeda K, Takemoto C, Kobayashi I, Watanabe A, Nobukuni Y, Fisher DE, et al. Ser298 of MITF, a mutation site in Waardenburg syndrome type 2, is a phosphorylation site with functional significance. Hum Mol Genet. 2000;9(1): 125-32.

38. Grill C, Bergsteinsdó ttir K, Ögmundsdó ttir MH, Pogenberg V, Schepsky A, Wilmanns $\mathrm{M}$, et al. MITF mutations associated with pigment deficiency syndromes and melanoma have different effects on protein function. Hum Mol Genet. 2013;22(21):4357-67.

39. Yan $X$, Zhang $T$, Wang $Z$, Jiang $Y$, Chen $Y$, Wang $H$, et al. A novel mutation in the MITF may be digenic with GJB2 mutations in a large Chinese family of Waardenburg syndrome type II. J Genet Genomics. 2011;38(12):585-91. https://doi.org/10.1016/j.jgg.2011.11.003.

40. Ren $\mathrm{S}$, Chen $\mathrm{X}$, Kong $\mathrm{X}$, Chen $\mathrm{Y}$, Wu Q, Jiao Z, et al. Identification of six novel variants in Waardenburg syndrome type II by next-generation sequencing. Mol Genet Genomic Med. 2020;8(3):1-8.

41. Kawakami A, Fisher DE. The master role of microphthalmia-associated transcription factor in melanocyte and melanoma biology. Lab Investig. 2017;97(6):649-56. https://doi.org/10.1038/labinvest.2017.9.

42. Udono T, Yasumoto Kl, Takeda K, Amae S, Watanabe Kl, Saito H, et al. Structural organization of the human microphthalmia-associated transcription factor gene containing four alternative promoters. Biochim Biophys Acta - Gene Struct Expr. 2000;1491(1-3):205-19.

43. Shibahara S. Microphthalmia-associated transcription factor (MITF): Multiplicity in structure, function, and regulation. J Investig Dermatology Symp Proc. 2001; 6(1):99-104. https://doi.org/10.1046/j.0022-202x.2001.00010.x.

44. Léger S, Balguerie X, Goldenberg A, Drouin-Garraud V, Cabot A, AmstutzMontadert l, et al. Novel and recurrent non-truncating mutations of the MITF basic domain: Genotypic and phenotypic variations in Waardenburg and Tietz syndromes. Eur J Hum Genet. 2012;20(5):584-7.

45. George A, Zand DJ, Hufnagel RB, Sharma R, Sergeev YV, Legare JM, et al. Biallelic mutations in MITF cause Coloboma, Osteopetrosis, Microphthalmia, Macrocephaly, Albinism, and Deafness. Am J Hum Genet. 2016;99(6):138894. https://doi.org/10.1016/j.ajhg.2016.11.004.

46. Bondurand N, Girard M, Pingault V, Lemort N, Dubourg O, Goossens M. Human Connexin 32, a gap junction protein altered in the X-linked form of Charcot-Marie-Tooth disease, is directly regulated by the transcription factor SOX10. Hum Mol Genet. 2001;10(24):2783-95.

47. Suzuki N, Mutai H, Miya F, Tsunoda T, Terashima H, Morimoto N, et al. A case report of reversible generalized seizures in a patient with Waardenburg syndrome associated with a novel nonsense mutation in the penultimate exon of SOX10. BMC Pediatr. 2018;18(1):2-5.

48. Yu Y, Liu W, Chen M, Yang Y, Yang Y, Hong E, et al. Two novel mutations of PAX3 and SOX10 were characterized as genetic causes of Waardenburg syndrome. Mol Genet Genomic Med. 2020;8(5):1-8.

49. Bondurand N, Dastot-Le Moal F, Stanchina L, Collot N, Baral V, Marlin S, et al. Deletions at the SOX10 gene locus cause Waardenburg syndrome types 2 and 4. Am J Hum Genet. 2007;81(6):1169-85.

50. Wang Q, Fang WH, Krupinski J, Kumar S, Slevin M, Kumar P. Pax genes in embryogenesis and oncogenesis. Genes J Cell Mol Med. 2008;12(6A): 2281-94. 
51. Boudjadi S, Chatterjee B, Sun W, Vemu P, Barr FG. The expression and function of PAX3 in development and disease. Gene. 2018;666(May):145-57. https://doi.org/10.1016/j.gene.2018.04.087.

52. Tassabehii M, Newton VE, Read AP. Waardenburg syndrome type 2 caused by mutations in the human microphthalmia (MITF) gene. Nat Genet. 1994; 8(3):251-5 http://www.nature.com/articles/ng1194-251.

53. Choi EY, Choi W, Lee CS. A novel PAX3 mutation in a Korean patient with Waardenburg syndrome type 1 and unilateral branch retinal vein and artery occlusion: A case report 11 Medical and Health Sciences 1113 Ophthalmology and Optometry. BMC Ophthalmol. 2018;18(1):4-9.

54. Richards S, Aziz N, Bale S, Bick D, Das S, Gastier-Foster J, et al. Standards and guidelines for the interpretation of sequence variants: A joint consensus recommendation of the American College of Medical Genetics and Genomics and the Association for Molecular Pathology. Genet Med. 2015; 17(5):405-24.

\section{Publisher's Note}

Springer Nature remains neutral with regard to jurisdictional claims in published maps and institutional affiliations.

Ready to submit your research? Choose BMC and benefit from:

- fast, convenient online submission

- thorough peer review by experienced researchers in your field

- rapid publication on acceptance

- support for research data, including large and complex data types

- gold Open Access which fosters wider collaboration and increased citations

- maximum visibility for your research: over $100 \mathrm{M}$ website views per year

At $\mathrm{BMC}$, research is always in progress.

Learn more biomedcentral.com/submissions 